\title{
Power control based on the Stackelberg game in two-tier femtocell networks
}

\author{
Zhixin Liu*, Shiyi Li, Lili Hao, Hongjiu Yang, Xinping Guan and Xinbin Li
}

\begin{abstract}
In this paper, the resource allocation strategy is investigated for a spectrum sharing two-tier femtocell networks, in which a central macrocell is underlaid with distributed femtocells. The spectral radius is introduced to address the conditions that any feasible set of users' signal-to-interference-plus-noise ratio requirements should satisfy in femtocell networks. To develop power allocation scheme with the derived conditions, a Stackelberg game is formulated, which aims at the utility maximization both of the macrocell user and femtocell users. The distributed power control algorithm is given to reduce the cross-tier interference between the macrocell and femtocell with same channel. At last, admission control algorithm is proposed, aiming to exploit the network resource effectively. Numerical results show that the proposed resource allocation schemes are effective in reducing power consumption and more suitable in the densely deployed scenario of the femtocell networks. Meanwhile, it also presents that the distributed power allocation scheme combined with admission control can protect the performance of all active femtocell users in a robust manner.
\end{abstract}

\section{Index Terms}

Femtocell, resource allocation, spectrum sharing, power control, Stackelberg game.

\section{INTRODUCTION}

In recent years, femtocell technology has been rapidly developed since it is able to extend cellular coverage, introduce new services and increase wireless network capacity [1]. Femtocell technology connects standard mobile devices to an operator's network using broadband connections via a third party backhaul (e.g. digital subscriber line (DSL) or cable modem). The co-channel spectrum sharing between femtocells and macrocells, together with the "random" placement of femtocells, may make the coexistence of macrocells and femtocells unsuccessful [2],[3]. Therefore, mitigating the cross-tier interference is one of the major challenges in femtocell networks [4]. At present, many approaches are applied to manage the interference in two-tier femtocell networks, such as power control, multiple antennas [5], adaptive femtocell base station (FBS) access scheme [6], soft radio technique [7] and spectrum allocation [8].

In this paper, we focus on the power allocation method to mitigate the interference. A distributed utility based on the signal-to-interference-plus-noise ratio (SINR) adaptation algorithm is proposed in [9] to alleviate the crosstier interference. In [10], the distributed power control algorithm is used to eventually achieve their fixed target SINRs. In [11], the distributed joint power control algorithm is proposed for the management of interference in two-tier femtocell networks. However, the literatures of [9], [10] and [11] only consider the power control algorithm to mitigate the interference and ignore the different requirements of users. In order to maximize their respective utility of femtocell users (FUEs) and macrocell user (MUE), the power control based on the Stackelberg game is proposed. In [12], a dual-utility Stackelberg game model is investigated, in which macrocell and femtocell have different utilities. In [13], a power allocation strategy based on interference pricing for a spectrum sharing femtocell network is investigated. However it puts forward high demand to the backhaul capacity due to price bargaining and transmission. A joint power control and resource allocation scheme for co-channel deployed femtocells in macrocell work is proposed to guarantee the quality of service (QoS) requirement of both MUE and FUEs in [14]. In [15], the authors propose the dynamic OFDMA subchannel assignment problem while jointly considering power assignment to mitigate the interference. The paper of [16] proposes a hierarchical power control algorithm with interference

* Corresponding author, Zhixin Liu, E-mail address: lzxauto@ysu.edu.cn. Zhixin Liu, Shiyi Li, Lili Hao, Hongjiu Yang and Xinbin Li are with the Institute of Electrical Engineering, Yanshan University, Qinhuangdao 066004, China. Email: 1zxauto@ysu.edu.cn, lisyysu@ 126.com; yanghongjiu@ysu.edu.cn; lixb@ysu.edu.cn. Xinping Guan is with the School of Electronic Information and Electrical Engineering, Shanghai Jiaotong University, Shanghai 200240, China. Email:xpguan@sjtu.edu.cn 
allowance in two-tier femtocell networks. The papers [14], [15] and [16] propose the power control algorithm to mitigate the interference. However, these schemes adopt the centralized algorithm. This method requires more channel information exchange. In [17], a SINR based power control scheme is presented to restrict the uplink interference. However, the study focuses on the sparse scenarios and it shows significant limitations in densely deployed case. Price-based resource allocation strategies based on Stackelberg game are investigated in [18] for two-tier femtocell networks. Nevertheless the interference price is adjusted manually which could not be flexible in the actual environment. In [19], three spectrum usage schemes together with the selection of the BSs are proposed to deal with the cross-tier interference. In [20], a hierarchical power control algorithm with interference allowance in two-tier heterogeneous networks is proposed. Specifically, a scenario where densely deployed femtocells exhibit on-off activity is considered. [21] considers the distributed Pareto-optimal joint optimization of SINR assignment and power control. There are some users who do not meet the communication requirements based on above power control algorithms. In order to improve the users' communication quality, the femtocell users whose QoS can not be guaranteed or result in serious interference to macrocell users, should be removed. So, in this paper, we propose the admission control algorithm. In [22], the Pareto-optimal power control algorithm are designed to jointly maximize the total utility of both classes of user under the constraint of the spectral radius. In our paper, the spectral radius will be used to analyze the relationship among the users' SINR in two-tier femtocell networks.

In this paper, we derive the conditions that any feasible set of users' SINR requirements should satisfy to quantify near-far effects. Based on the analysis results, two different resource allocation strategies are proposed. If the set of SINRs is feasible, we propose a power allocation scheme to minimize the power consumption. In the case that not all the SINR requirements of the users can be satisfied simultaneously, the price-based interference management scheme is proposed. It integrates the utility optimization and restricts the cross-tier interference at the MBS below a given threshold. In particular, the interference price and the co-tier interference are adjusted adaptively which is different from the pricing scheme proposed in [17] and [18]. Then, the distributed power allocation scheme is developed based on Stackelberg game. Different from [12] and [13], our work just requires smaller channel capacity and limited information to exchange. At last, in order to utilize the system resource more reasonably and efficiently, admission control is introduced to two-tier femtocell networks. Simulation results give the evidence that the proposed power allocation method can efficiently reduce the power consumption,especially when there are large number of FUEs. The main contributions of this paper are summarized as follows:

- We propose a method of Stackelberg game in order to achieve the optimal power control strategy. Two different utility functions are designed for femtocell users (FUEs) and macrocell user (MUE), that can satisfy respective requirement by maximizing their own utility.

- The price-based interference management scheme is introduced to mitigate the interference, which can protect the benefit the MUE and optimize the power.

- A distributed power allocation algorithm with low complexity is proposed to reduce information exchange, and a admission power control is introduced to utilize the system resource more reasonably and efficiently.

The rest of the paper is organized as follows. Section II presents the system model. Section III describes the problem formulation. Then, the Stackelberg game analysis is presented in IV. Numerical results are given in section $\mathrm{V}$, which follows the conclusion in section VI.

\section{SYSTEM MODEL}

We consider the two-tier femtocell network with the model of single cell WCDMA system. The network consists of one central macrocell and $N$ randomly distributed femtocells. Fig. 1 gives the description of uplink transmission in two-tier femtocell networks. As shown in Fig. 1, the MBS serves in a region with radius $R_{c}$. The red and black dashed lines respectively represent the uplink interference from the macrocell user and FUEs. We assume that there is one corresponding FBS providing service in each femtocell. Besides, orthogonal uplink signaling is assumed in each slot, where a given time slot is considered. The channel gains $g_{i, j}$ as in the IMT-2000 specification are 
presented using simplified path loss model[23].

$$
g_{i, j}=\left\{\begin{array}{cc}
K_{c} \min \left(D^{-\alpha_{c}}, 1\right), & i=j=0 \\
K_{f i} R_{f}^{-\alpha_{\beta}}, & i=j \\
K_{f o} W \min \left(D_{0, j}^{-\alpha_{f o}}, 1\right), & i=0, j>0 \\
K_{c} \min \left(D_{i, j}^{-\alpha_{c}}, 1\right), & i>0, j=0 \\
K_{f o} W^{2} \min \left(D_{i, j}^{-\alpha_{f o}}, 1\right), & i \neq j \neq 0 .
\end{array}\right.
$$

In (1), $\alpha_{c}, \alpha_{\beta}, \alpha_{f o}$ denote the macrocell, indoor and indoor to outdoor femtocell path loss exponents, respectively. $D_{i, j}$ is the distance between user $j$ and BS $i$. Noting that $i=0$ and $j=0$ respectively denote the MBS and the MUE. $D$ is the distance between MUE to MBS. Defining $f_{c}$ as the carrier frequency in MHz, $K_{c}=30 \log _{10} f_{c}-71 d B$ equals the fixed decibel propagation loss during the transmission from the MUE to the MBS. The term $K_{f i}$ is the fixed loss between FUE $i$ and its corresponding FBS. $K_{f o}$ is defined as the fixed loss between FUE $i$ to a different FBS and the assumption $K_{f o}=K_{c}$ is given. The term $W$ explicitly models partition loss during indoor-outdoor transmission to the MBS.

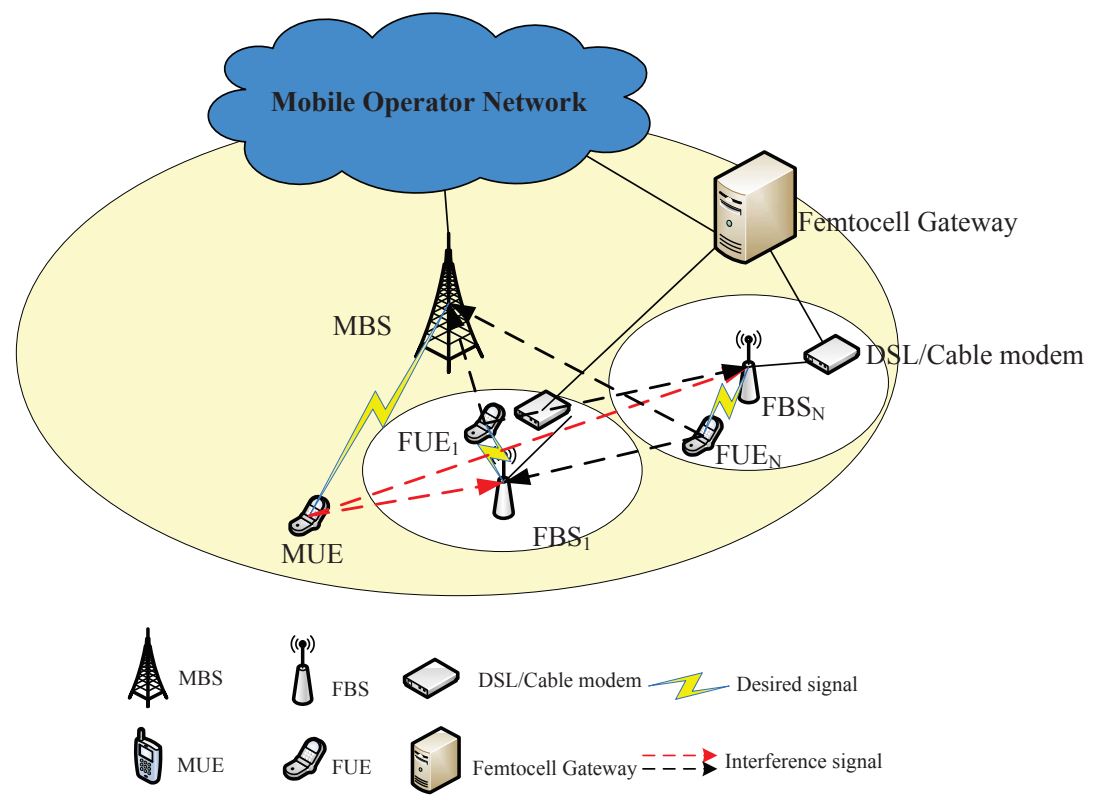

Fig. 1. System model.

In this paper, CDMA technology is adopted in the Femtocell networks. Let $i \in\{0,1 \cdots N\}$ denote the user set connected to the corresponding BS. The background noise is additive White Gaussian Noise with power spectral density $\eta_{0}$. The SINR of user $i$ is given as

$$
\gamma_{i}=\frac{W}{R} \frac{g_{i, i} p_{i}}{\sum_{j=0, j \neq i}^{N} g_{i, j} p_{j}+W \eta_{0}},
$$

where $W$ is the bandwidth and $R$ is the transmission rate. Define $L$ as the processing gain, i.e. $L=W / R$. In our paper, we assume that the value of $L$ is 128. $p_{i}$ is the transmission power of FUE $i$. The term $\sum_{j=0, j \neq i}^{N} g_{i, j} p_{j}$ is the total interference caused by other users. Denote $\delta^{2}=W \eta_{0}$ as the noise. We define the normalized SINR as

$$
\bar{\gamma}_{i}=\frac{\gamma_{i}}{L}
$$


In the transmission process, a set of minimum SINRs should be supported, i.e.,

$$
\Gamma_{i} \leq \bar{\gamma}_{i}=\frac{g_{i, i} p_{i}}{\sum_{j=0, j \neq i}^{N} g_{i, j} p_{j}+\delta^{2}}
$$

where $\Gamma_{i}$ denotes the minimum required SINR of FUE $i$. Inequality (4) can also be formulated as

$$
\mathbf{p} \geq \boldsymbol{\Gamma} \mathbf{G p}+\boldsymbol{\eta}, \mathbf{p} \geq 0,
$$

where $\boldsymbol{\Gamma}=\operatorname{diag}\left(\Gamma_{0}, \Gamma_{1}, \cdots, \Gamma_{N}\right), \boldsymbol{\eta}=\left(\eta_{0}, \eta_{1}, \cdots, \eta_{N}\right)=\left(\frac{\delta^{2}}{g_{0,0}}, \frac{\delta^{2}}{g_{1,1}}, \cdots, \frac{\delta^{2}}{g_{N, N}}\right), \boldsymbol{\Gamma}$ is the normalized noise power vector. $\mathbf{p}=\left(p_{0}, p_{1}, \cdots, p_{N}\right)$ is power vector of users. $\mathbf{G}$, as the normalized channel gain matrix, is defined as follows.

$$
G_{i, j}=\left\{\begin{array}{cc}
\frac{g_{i, j}}{g_{i, i}}, & i \neq j \\
0, & i=j .
\end{array}\right.
$$

If there exist feasible solution satisfying the inequation (5), all the users involved in the network can achieve the desired SINR requirements[22].

To get the feasible solution of (5), the concept of spectral radius of matrix is introduced. Let $\rho(\boldsymbol{M})$ denote the spectral radius of matrix $M$, that is, its maximum modulus eigenvalue. Matrix $M$ can be written as follows

$$
\boldsymbol{M}=\left[\begin{array}{cccc}
M_{11} & M_{12} & \cdots & M_{1 n} \\
M_{21} & M_{22} & \cdots & M_{2 n} \\
\vdots & \vdots & \ddots & \vdots \\
M_{n 1} & M_{n 2} & \cdots & M_{n n}
\end{array}\right]
$$

For a given index $i, M_{i}$ denotes the principal submatrix of $\boldsymbol{M}$ by removing the elements of the $i^{\text {th }}$ row and the $i^{\text {th }}$ column of $\boldsymbol{M}$. Let $M_{i *}$ present the $i^{t h}$ row of blocks with the $i^{\text {th }}$ element $M_{i i}$ removed. Moreover, $M_{* i}$ presents the $i^{t h}$ column of blocks with the $i^{t h}$ element $M_{i i}$ removed. According to the definition of Perron complement, the spectral radius of $M$ satisfies

$$
\rho(\boldsymbol{M})=M_{i i}+M_{i *}\left(\rho I-M_{i}\right)^{-1} M_{* i}
$$

To guarantee the existence of a feasible power vector $\mathbf{p}>0$, the condition $\rho(\boldsymbol{\Gamma} \boldsymbol{G})<1$ should be satisfied [23]. From equation (5), we can get that

$$
\mathbf{p} \geq(\boldsymbol{I}-\boldsymbol{\Gamma} \boldsymbol{G})^{-1} \boldsymbol{\eta}
$$

Through the Karush-Kuhn-Tucker (KKT) conditions, we know that when $\mathbf{p}=(\boldsymbol{I}-\boldsymbol{\Gamma} \boldsymbol{G})^{-1} \boldsymbol{\eta}$ can obtain the optimal power. Then we have

$$
\mathbf{p}^{*}=(\boldsymbol{I}-\boldsymbol{\Gamma} \boldsymbol{G})^{-1} \boldsymbol{\eta}
$$

is a solution of (5). Moreover, any other $\mathbf{p}$ satisfying (5) would require much power from every transmitter, i.e.

$$
\mathbf{p} \geq \mathbf{p}^{*}=(\boldsymbol{I}-\boldsymbol{\Gamma} \boldsymbol{G})^{-1} \boldsymbol{\eta} .
$$

In the actual transmission, a larger SINR requirement of the user is usually set to avoid the quality falling caused by the sudden access of the users. Here, we define $\Gamma_{c}=\Gamma_{0}$ and $\boldsymbol{\Gamma}_{f}=\operatorname{diag}\left(\Gamma_{1}, \Gamma_{2}, \cdots, \Gamma_{N}\right)$, and define $\boldsymbol{G}_{f}$ as the normalized channel gain matrix whose elements are the channel gains between each femtocell and its surrounding cochannel femtocells. Besides, we define $\boldsymbol{q}_{c}^{T}=\left(G_{01}, \cdots, G_{0 N}\right)$ as the normalized channel gains from FUEs to the MBS. Similarly, $\boldsymbol{q}_{f}^{T}=\left(G_{10}, \cdots, G_{N 0}\right)$ is the normalized channel gains from the MUE to its surrounding FBSs. Then, matrix $\Gamma G$ can be expressed as

$$
\boldsymbol{\Gamma} \boldsymbol{G}=\left[\begin{array}{cc}
0 & \Gamma_{c} \boldsymbol{q}_{c}^{T} \\
\boldsymbol{\Gamma}_{f} \boldsymbol{q}_{f} & \boldsymbol{\Gamma}_{f} \boldsymbol{G}_{f}
\end{array}\right]
$$

To enhance the adaptability, it is assumed that $\Gamma_{i}$ is not strictly greater than the predefined SINR requirement of FUE $i$, i.e., $\bar{\rho}=1-\epsilon(\epsilon>0$, which is a very small constant). 
As $\bar{\rho} \rightarrow 1$, the optimal resource allocation scheme can be found. We can get the optimal solution which satisfies

$$
\rho(\boldsymbol{\Gamma} \boldsymbol{G})<\bar{\rho} .
$$

The power allocation strategy is set by $\mathbf{p}^{*}=(\boldsymbol{I}-\boldsymbol{\Gamma} \boldsymbol{G})^{-1} \boldsymbol{\eta}$ to minimize the power consumption, if it is possible to satisfy the SINR requirements for all links simultaneously. Moreover, in order to quantify near-far effects with universal frequency reuse, we derive the conditions that any feasible set of users' SINR requirements should satisfy.

Lemma 1: [28, Horn, Corollary 8.1.20] $\boldsymbol{M}_{n}$ is defined as a $n \times n$ matrix. Let $\boldsymbol{A}, \boldsymbol{B} \in \boldsymbol{M}_{n}$. If $0 \leq \boldsymbol{A} \leq \boldsymbol{B}$, then $\rho(\boldsymbol{A}) \leq \rho(\boldsymbol{B})$.

Theorem 1: If there exists a feasible matrix of all users' required SINRs $\boldsymbol{\Gamma}$ and $\rho(\boldsymbol{\Gamma} \boldsymbol{G})=\nu$. A constraint can be given as follows to get a feasible set of SINRs.

$$
\boldsymbol{\Gamma}=\left\{\left(\Gamma_{c}, \boldsymbol{\Gamma}_{f}\right): 0 \leq \boldsymbol{\Gamma}_{f} \leq \frac{1}{\rho\left(\boldsymbol{G}_{f}\right)}, \Gamma_{c}=\frac{\nu^{2}}{\boldsymbol{q}_{c}^{T}\left[\boldsymbol{I}-\left(\boldsymbol{\Gamma}_{f} / \nu\right) \boldsymbol{G}_{f}\right]^{-1} \boldsymbol{q}_{f}}\right\} .
$$

Proof: Considering $\boldsymbol{\Gamma}_{f} \boldsymbol{G}_{f}$ is a part of $\boldsymbol{\Gamma} \boldsymbol{G}$, the condition $\boldsymbol{\Gamma}_{f} \rho\left(\boldsymbol{G}_{f}\right)<\rho(\boldsymbol{\Gamma} \boldsymbol{G})$ can be verified by Lemma 1. We have $\boldsymbol{\Gamma}_{f} \rho\left(\boldsymbol{G}_{f}\right)<\nu<1$, then we can get the relationship as follows

$$
0 \leq \boldsymbol{\Gamma}_{f} \leq \frac{1}{\rho\left(\boldsymbol{G}_{f}\right)}
$$

According to equation (8), we can get

$$
\nu=0+\Gamma_{c} \boldsymbol{q}_{c}^{T}\left[\nu \boldsymbol{I}-\boldsymbol{\Gamma}_{f} \boldsymbol{G}_{f}\right]^{-1} \boldsymbol{\Gamma}_{f} \boldsymbol{q}_{f} .
$$

Since $\rho\left(\boldsymbol{\Gamma}_{f} \boldsymbol{G}_{f}\right)<\nu<1$, according to the literature [24], the inversion of matrix $\left[\boldsymbol{I}-\left(\boldsymbol{\Gamma}_{f} / \nu\right) \boldsymbol{G}_{f}\right]$ exists, and it is nonnegative. From (16),we can get

$$
\Gamma_{c}=\frac{\nu^{2}}{\boldsymbol{q}_{c}^{T}\left[\boldsymbol{I}-\left(\boldsymbol{\Gamma}_{f} / \nu\right) \boldsymbol{G}_{f}\right]^{-1} \boldsymbol{q}_{f}}
$$

The proof is completed.

Theorem 2: With a feasible set of SINR thresholds $\boldsymbol{\Gamma}_{f}$ and the constraint $\rho\left(\boldsymbol{\Gamma}_{f} \boldsymbol{G}_{f}\right)<1$, a necessary condition for MBS to set required SINRs could be described as $\Gamma_{c} \leq \frac{1}{\boldsymbol{q}_{c}^{T} \boldsymbol{\Gamma}_{f} \boldsymbol{q}_{f}}$. In order to ensure the SINR requirements of FUEs are positive, the following inequality should be satisfied.

$$
\Gamma_{c} \boldsymbol{\Gamma}_{f} \leq \frac{1}{\boldsymbol{q}_{c}^{T} \boldsymbol{q}_{f}}
$$

Proof: Applying the literature [28, Horn, Corollary 8.1.19], we can obtain

$$
\nu=\rho\left(\boldsymbol{\Gamma}_{f} \boldsymbol{G}_{f}+\boldsymbol{\Gamma}_{f} q_{f} \Gamma_{c} \boldsymbol{q}_{c}^{T} / \nu\right)
$$

by the derivation. Then, the following formula

$$
\rho\left(\boldsymbol{\Gamma}_{f} \boldsymbol{G}_{f}+\boldsymbol{\Gamma}_{f} q_{f} \Gamma_{c} \boldsymbol{q}_{c}^{T} / \nu\right) \geq \rho\left(\boldsymbol{\Gamma}_{f} q_{f} \Gamma_{c} \boldsymbol{q}_{c}^{T} / \nu\right)
$$

can be obtained based on Lemma 1. Applying the conclusion $\rho\left(\boldsymbol{q}_{f} \boldsymbol{q}_{c}^{T}\right)=\boldsymbol{q}_{c}^{T} \boldsymbol{q}_{f}$ [23], and considering the condition $\nu^{2} \leq 1$, we have

$$
\frac{\rho\left(\boldsymbol{\Gamma}_{f} q_{f} \Gamma_{c} \boldsymbol{q}_{c}^{T}\right)}{\nu} \leq \nu
$$

Then, it follows

$$
\Gamma_{c} \boldsymbol{\Gamma}_{f} \leq \frac{1}{\boldsymbol{q}_{c}^{T} \boldsymbol{q}_{f}}
$$

Theorem 2 is proved.

The given results in the above discussions are conducive to understand the connection between the users' SINR requirements. The reasonable requirements of SINR are necessary to keep the coexistence of all users. However, 
for the selfishness and distributed organisation of users, in order to pursue the maximum SINR and improve the benefit are usually the primary objective. How to get the balance of individual benefit and whole benefit is to be considered. Combining with the analysis of the spectral radius and SINR constraints, we propose a power allocation strategy to reduce the energy consumption and achieve the equilibrium of involved users.

\section{PROBLEM FORMULATION}

In the actual wireless network environment, it is difficult to satisfy all SINR requirements of the FUEs simultaneously. Hence, the scenario of $\rho(\boldsymbol{\Gamma} \boldsymbol{G})>\bar{\rho}$ is mainly considered. In the following section, the Stackelberg game formulation is presented to get feasible power allocation solutions.

\section{A. Utility Formulations}

In the Stackelberg game, the MBS as the leader protects itself by pricing the interference coming from femtocells and maximizes its utility function. Therefore, it is a strategy by selling the interference quota to FUEs to achieve the revenue. The revenue of the MBS is defined as

$$
U_{M B S}\left(c, \mathbf{p}^{\prime}\right)=c \sum_{i=1}^{N} p_{i} g_{0, i},
$$

where $c$ is the interference price, vector $\mathbf{p}^{\prime}=\left[p_{1}, p_{2}, \cdots, p_{N}\right]$ denotes the power levels for FUEs. It is worth emphasizing that the level of $c$ presents the willingness of FUE $i$ to buy the interference quota in price set by the MBS. To guarantee the communication quality of MUE, the SINR of MUE should be greater than the minimum SINR. The optimization problem of the MBS can be described as

$$
\begin{gathered}
\text { Problem 3.1 } \max _{c>0} U_{M B S}\left(c, \mathbf{p}^{\prime}\right)=c \sum_{i=1}^{N} p_{i} g_{0, i} \\
\text { s.t. } \\
\qquad \gamma_{0}=\frac{p_{0} g_{0,0}}{\sum_{i=1}^{N} p_{i} g_{0, i}+\delta^{2}} \geq \Gamma_{0},
\end{gathered}
$$

where $p_{0}$ is the MUE's transmission power. The term $\Gamma_{0}$ is the required SINR of the MBS. To protect the MBS's SINR, let

$$
Q=\frac{p_{0} g_{0,0}}{\Gamma_{0}}-\delta^{2}
$$

where $p_{0}$ and $\Gamma_{0}$ are all set as constants. Problem 3.1 can be rewritten as

$$
\text { Problem 3.2: } \max _{c>0} U_{M B S}\left(c, \mathbf{p}^{\prime}\right)=c \sum_{i=1}^{N} p_{i} g_{0, i}
$$

s.t.

$$
\sum_{i=1}^{N} p_{i} g_{0, i} \leq Q .
$$

For FUEs, the SINR logarithmic form is selected as the reward function. Besides, a cost function is also considered. Mathematically, we define the optimization problem of FUE $i$ as

$$
\text { Problem } 3.3: \max U_{i}\left(p_{i}, \mathbf{p}_{-i}^{\prime}\right)=\lambda_{i} \ln \left(1+\gamma_{i}\right)-c p_{i} g_{0, i},
$$

s.t.

$$
0 \leq p_{i} \leq p_{\max }
$$

where $\mathbf{p}_{-i}^{\prime}=\left[p_{1}, \cdots, p_{i-1}, p_{i+1}, \cdots, p_{N}\right]$ and $\lambda_{i}$ is the utility gain. It can be observed that adjusting $c$ will impact the non-cooperative game between the FUEs. With the increasing of the transmission power, the transmission rate 
will increase. Meanwhile, more interference to the MBS will be caused. To guarantee communication quality of the MUE, the MBS will increase the interference price $c$. This result will increase the cost of FUEs. Therefore, cost function is needed for FUE to maximize the corresponding utility.

\section{Stackelberg Game Analysis}

In this section, first, the Stackelberg equilibrium (SE) for the proposed game is defined. Second, we prove the existence and uniqueness of the subgame's Nash equilibrium (NE). Moreover, the solution of the Stackelberg game is given. Finally, a distributed power control algorithm combined with the admission control is provided.

\section{A. Stackelberg Game Equilibrium}

Stackelberg game is a strategic game that consists of a leader (MBS) and several followers (FUEs) competing for limited resources. All FUEs, competing each other in a non-cooperative manner to maximize their own utilities, form a lower subgame, $G=\left\{S,\left\{P_{i}\right\},\left\{U_{i}\right\}\right\}$, where $S$ is the player set, $\left\{P_{i}\right\}$ and $\left\{U_{i}\right\}$ are the strategy space and the utility of FUE $i$, respectively. In this part, the objective is to find the SE point. With the respective strategy, the MUE and all FUEs have no motivations to deviate it. The SE is defined as follows:

$$
U_{M B S}\left(c^{*}, \mathbf{p}^{\prime *}\right) \geq U_{M B S}\left(c, \mathbf{p}^{\prime}\right)
$$

and

$$
U_{i}\left(p_{i}^{*}, \mathbf{p}_{-i}^{*}\right) \geq U_{i}\left(p_{i}, \mathbf{p}_{-i}^{\prime}\right), \forall i \in S,
$$

where $c^{*}$ denotes the optimal price that can be obtained by maximizing the MBS's utility. $\mathbf{p}^{\prime *}=\left[p_{1}^{*}, p_{2}^{*}, \cdots, p_{N}^{*}\right]^{T}$ are the optimal power allocations for FUEs. The SE point $\left(c^{*}, \mathbf{p}^{\prime *}\right)$ can be obtained with any initial $\left(c, \mathbf{p}^{\prime}\right)$, if $c>0$ and $\mathbf{p}^{\prime}>0$.

Generally, we can obtain SE by finding the NE of its subgames. NE is defined that no player can unilaterally increase his own utility when all the other players do not change their strategies. It is because that adjusting the strategy will sacrifice the corresponding player's utility. Therefore, every rational FUE has no willingness to change the strategy individually in the NE point. In the macro network since there is only one user, getting the best response is not difficult. Therefore, the SE can be obtained by the following approach: firstly, FUEs maximize their utility functions for optimal powers with any given interference price. Then with the obtained best response, we solve the problem of the MBS to get the optimal interference price $c^{*}$. The existence of SE will be discussed in the following theorem.

Theorem 3: The SE exists in the Stackelberg game.

Proof: For the lower subgame, we have

1) In the one-dimensional space, convex set is a single point or a continuous line. For the strategy $p_{i} \in\left[0, p_{\max }\right]$, it means that $p_{i}$ is a convex and compact subset.

2) The derivative of $U_{i}\left(p_{i}, \mathbf{p}_{-i}\right)$ is given as follows

$$
\begin{aligned}
\frac{\partial U_{i}}{\partial p_{i}} & =\frac{\lambda_{i}}{1+\gamma_{i}\left(p_{i}, \mathbf{p}_{-i}^{\prime}\right)} \cdot \frac{\gamma_{i}\left(p_{i}, \mathbf{p}_{-i}^{\prime}\right)}{p_{i}}-c g_{0, i} \\
& =\frac{L \lambda_{i} g_{i, i}}{I_{i}\left(\mathbf{p}_{-i}^{\prime}\right)+L p_{i} g_{i, i}}-c g_{0, i},
\end{aligned}
$$

where $I_{i}\left(\mathbf{p}_{-i}^{\prime}\right)=\sum_{j \neq i} p_{j} g_{i, j}+\delta^{\prime 2}, i, j \in S$ and $\delta^{\prime 2}$ is the background noise at the FBS $i$ taking into account the additional interference from the MUE. We assume that $\lambda_{i} \neq 0$, the second derivative of $U_{i}$ can be given as

$$
\frac{\partial^{2} U_{i}}{\partial p_{i}^{2}}=-\frac{L^{2} \lambda_{i} g_{i, i}^{2}}{\left(I_{i}\left(\mathbf{p}_{-i}^{\prime}\right)+L p_{i} g_{i, i}\right)^{2}}<0 .
$$

Obviously, the revenue function $U_{i}$ is concave in $p_{i}$. If the above conditions are satisfied, the NE exists in the lower subgame [25]. Therefore, the SE exists in the Stackelberg game. 


\section{B. Solution to the Stackelberg Game}

In the subsection, we focus on obtaining the optimal strategies in the game. The best responses of the FUEs will be presented, followed by the MBS's best response.

1) FUEs' best responses: It has been proved that the utility $U_{i}\left(p_{i}, \mathbf{p}_{-i}^{\prime}\right)$ is concave in $p_{i}$. The best response of the FUE $i$ can be obtained when the first derivative of function $U_{i}\left(p_{i}, \mathbf{p}_{-i}^{\prime}\right)$ equals zero, i.e.,

$$
\frac{\partial U_{i}}{\partial p_{i}}=\frac{\lambda_{i}}{1+\gamma_{i}\left(p_{i}, \mathbf{p}_{-i}^{\prime}\right)} \cdot \frac{\gamma_{i}\left(p_{i}, \mathbf{p}_{-i}^{\prime}\right)}{p_{i}}-c g_{0, i}=0 .
$$

In the case, the best response can be derived as follows

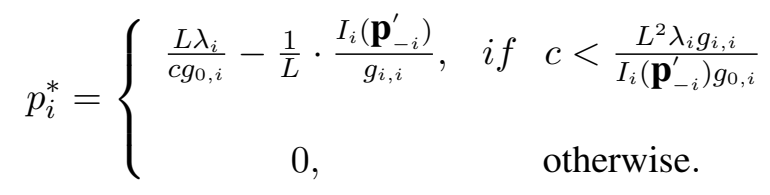

The optimal solution of FUE $i$ can be rewritten as

$$
p_{i}^{*}+\frac{1}{L} \cdot \frac{\sum_{j=1, j \neq i}^{N} p_{j} g_{i, j}}{g_{i, i}}=\frac{L \lambda_{i}}{c g_{0, i}}-\frac{1}{L} \cdot \frac{\delta^{\prime 2}}{g_{i, i}}, \forall i \in S .
$$

We can get the matrix form

$$
\boldsymbol{G}_{f} \mathbf{p}^{*}=\frac{\boldsymbol{A}}{c}-\boldsymbol{B}
$$

where

$$
\boldsymbol{A}=\left[\begin{array}{c}
\frac{L \lambda_{1}}{g_{0,1}} \\
\frac{L \lambda_{2}}{g_{0,2}} \\
\vdots \\
\frac{L \lambda_{N}}{g_{0, N}}
\end{array}\right], \boldsymbol{B}=\frac{1}{L}\left[\begin{array}{c}
\frac{\delta^{\prime 2}}{g_{1,1}} \\
\frac{\delta^{\prime 2}}{g_{2,2}} \\
\vdots \\
\frac{\delta^{\prime 2}}{g_{N, N}}
\end{array}\right], \boldsymbol{G}_{f}=\frac{1}{L}\left[\begin{array}{cccc}
1 & \frac{g_{1,2}}{g_{1,1}} & \cdots & \frac{g_{1, N}}{g_{1,1}} \\
\frac{g_{2,1}}{g_{2,2}} & 1 & \cdots & \frac{g_{2, N}}{g_{2,2}} \\
\vdots & \vdots & \ddots & \vdots \\
\frac{g_{N, 1}}{g_{N, N}} & \frac{g_{N, 2}}{g_{N, N}} & \cdots & 1
\end{array}\right]
$$

Assuming that the matrices of $\boldsymbol{G}_{f}$ in the above formula is invertible. The optimal value of $\mathbf{p}^{*}$ for the non-cooperative game can be described as

$$
\mathbf{p}^{\prime *}=\frac{\boldsymbol{G}_{f}^{-1} \boldsymbol{A}}{c}-\boldsymbol{G}_{f}^{-1} \boldsymbol{B},
$$

2) MBS's best response: Substituting (29) into (22), we can get

$$
\begin{aligned}
& \max _{c>0} U_{M B S}\left(c, \mathbf{p}^{\prime}\right)=\boldsymbol{G}_{0} \boldsymbol{G}_{f}^{-1} \boldsymbol{A}-c \boldsymbol{G}_{0} \boldsymbol{G}_{f}^{-1} \boldsymbol{B}, \\
\text { s.t. } & \frac{\boldsymbol{G}_{0} \boldsymbol{G}_{f}^{-1} \boldsymbol{A}}{c}-\boldsymbol{G}_{0} \boldsymbol{G}_{f}^{-1} \boldsymbol{B} \leq Q,
\end{aligned}
$$

where $\boldsymbol{G}_{0}=\left[g_{01}, g_{02}, \cdots, g_{0 N}\right]$. Obviously, $\boldsymbol{G}_{0} \boldsymbol{G}_{f}^{-1} \boldsymbol{A}$ is a constant. Problem 3.2 can be eventually simplified as

$$
\begin{aligned}
\text { Problem 3.4: } & \min _{c>0} c \boldsymbol{G}_{0} \boldsymbol{G}_{f}^{-1} \boldsymbol{B} \\
\text { s.t. } & \frac{\boldsymbol{G}_{0} \boldsymbol{G}_{f}^{-1} \boldsymbol{A}}{c}-\boldsymbol{G}_{0} \boldsymbol{G}_{f}^{-1} \boldsymbol{B} \leq Q .
\end{aligned}
$$

It is obvious that $c \boldsymbol{G}_{0} \boldsymbol{G}_{f}^{-1} \boldsymbol{B}$ is concave in $c$. Thus Problem 3.4 is a convex optimization problem. The KarushKuhn-Tucker (KKT) conditions must be satisfied for the optimal solution. Consequently, we construct the Lagrange function as

$$
L(c, \alpha, \beta)=c \boldsymbol{G}_{0} \boldsymbol{G}_{f}^{-1} \boldsymbol{B}+\alpha\left(\frac{\boldsymbol{G}_{0} \boldsymbol{G}_{f}^{-1} \boldsymbol{A}}{c}-\boldsymbol{G}_{0} \boldsymbol{G}_{f}^{-1} \boldsymbol{B}-Q\right)+\beta c
$$


where $c \geq 0, \alpha \geq 0$ and $\beta \geq 0$. Besides, the KKT conditions as follows should also be involved

$$
\begin{gathered}
\frac{\partial L(c, \alpha, \beta)}{\partial c}=\boldsymbol{G}_{0} \boldsymbol{G}_{f}^{-1} \boldsymbol{B}-\frac{\alpha}{c^{2}} \boldsymbol{G}_{0} \boldsymbol{G}_{f}^{-1} \boldsymbol{A}+\beta=0, \\
\alpha\left(\frac{\boldsymbol{G}_{0} \boldsymbol{G}_{f}^{-1} \boldsymbol{A}}{c}-\boldsymbol{G}_{0} \boldsymbol{G}_{f}^{-1} \boldsymbol{B}-Q\right)=0, \\
\beta c=0 .
\end{gathered}
$$

From equation (33), we can get

$$
c^{2}=\frac{\alpha \boldsymbol{G}_{0} \boldsymbol{G}_{f}^{-1} \boldsymbol{A}}{\boldsymbol{G}_{0} \boldsymbol{G}_{f}^{-1} \boldsymbol{B}+\beta} .
$$

If $\alpha=0$, we have $c=0$ which contradicts with the inequality

$$
\frac{\boldsymbol{G}_{0} \boldsymbol{G}_{f}^{-1} \boldsymbol{A}}{c}-\boldsymbol{G}_{0} \boldsymbol{G}_{f}^{-1} \boldsymbol{B} \leq Q .
$$

Therefore, the optimal interference price $c^{*}$ can be derived from (34), described as

$$
c^{*}=\frac{\boldsymbol{G}_{0} \boldsymbol{G}_{f}^{-1} \boldsymbol{A}}{\boldsymbol{G}_{0} \boldsymbol{G}_{f}^{-1} \boldsymbol{B}+Q} .
$$

We can obtain the best solution of the proposed problem which is formulated as the point $\left(c^{*}, \mathbf{p}^{*}\right)$.

\section{Distributed Power Control Algorithm and Admission Control}

In two-tier femtocell networks, a distributed iterative power update rule is designed to reduce the information exchange. For the optimal interference price $c$, the power control strategy can be obtained as follows,

$$
\begin{aligned}
p_{i} & =\frac{L \lambda_{i}}{c g_{0, i}}-\frac{1}{L} \cdot \frac{I_{i}\left(\mathbf{p}_{-i}^{\prime}\right)}{g_{i, i}} \\
& =\frac{1}{g_{i, i}}\left(a_{i}-\frac{\sum_{j=1, j \neq i}^{N} y_{j}}{L}\right),
\end{aligned}
$$

where $a_{i}=\frac{L \lambda_{i} g_{i, i}}{c g_{0, i}}$. Considering the transmission power $p_{i}$ should be limited within $\left[0, p_{\max }\right]$, we rewrite the iteration strategy as

$$
p_{i}^{(k+1)}=\min \left\{\left[\frac{1}{g_{i, i}}\left(a_{i}-\frac{\sum_{j=1, j \neq i}^{N} y_{j}^{(k)}}{L}\right)\right]^{+}, p_{\max }\right\}, \forall i \in S,
$$

where $y_{j}=p_{j} g_{i, j} \cdot y_{j}^{(k)}$ denotes the interference to FBS $i$ introduced by the transmission power of FUE $j$ at time $k$. It can be seen from the expression of the power update rule that the algorithm can lend itself to a distributed implementation. In the iterative process, each user only needs to exchange the received interference power and its own power channel gain. It can be seen that the distributed power control algorithm can greatly reduce the information exchange.

Theorem 4: The distributed power control algorithm proposed is globally stable, and converges to the unique equilibrium solution from any feasible staring point, if the following condition is satisfied

$$
\frac{N-1}{L}<1
$$

Proof: Define $\Delta y_{i}^{(k)}=y_{i}^{(k)}-y_{i}^{*}$ and $\bar{y}_{-i}^{(k)}=\sum_{j=1, j \neq i}^{N} y_{j}^{(k)}$, where $y_{i}^{*}$ is the power of the FBS $i$ obtained at 
the SE point. First, we consider the case that $y_{i}^{*}=0, \forall i$, then we can obtain that

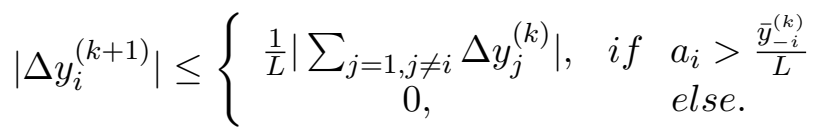

If $y_{i}^{*}>0$, given all FUEs' transmission powers except FUE $i$ at time $k, y_{j}^{(k)}(j \neq i)$, we have

$$
\left|\Delta y_{i}^{(k+1)}\right|\left\{\begin{array}{lll}
<\frac{1}{L}\left|\sum_{j=1, j \neq i} \Delta y_{j}^{(k)}\right|, & \text { if } & a_{i}>\frac{\bar{y}_{-i}^{(k)}}{L} \\
=\frac{1}{L}\left|\sum_{j=1, j \neq i} \Delta y_{j}^{(k)}\right|, & \text { else, }
\end{array}\right.
$$

i.e.,

$$
\left|\Delta y_{j}^{(k+1)}\right| \leq \frac{1}{L}\left|\sum_{j=1, j \neq i} \Delta y_{j}^{(k)}\right| .
$$

It is observed that the inequality (43) holds for any FUE. The $l_{\infty}$-norm of vector $\triangle \mathbf{y}$ over the player set $S$ is denoted by $\|\triangle \mathbf{y}\|_{S}$, i.e., $\|\triangle \mathbf{y}\|_{\infty}=\max _{i \in S}\left|\triangle y_{i}\right|$. From (43), we have

$$
\left\|\Delta y^{(k+1)}\right\|_{\infty} \leq \frac{1}{L} \max _{i} \sum_{j=1, j \neq i}^{N}\left|\Delta y_{j}\right|^{(k)} \leq \frac{N-1}{L}\left\|\Delta y^{(k)}\right\|_{\infty} .
$$

Remark 1: Note that the proposed power control strategy with the maximum power constraint still converges to a unique NE point. It is suggested that $A\left(p_{i}\right)=p_{\max }$ is a standard interference function with $p_{\max } \geq 0$, if it meets positivity, monotonicity, and scalability. In the following, a brief proof is given.

Let $\left.A\left(p_{i}\right)=p_{\max } .1\right)$ Positivity: Since $p_{\max }>0$, then $A\left(p_{i}\right)>0$. 2) Monotonicity: when $p_{i}^{\prime}>p_{i}$, we have $\left.A\left(p_{i}^{\prime}\right)=p_{\max }=A\left(p_{i}\right) .3\right)$ Scalability: $\forall \theta>1, \theta A\left(p_{i}\right)=\theta p_{\max }=\theta A\left(\theta p_{i}\right)>A\left(\theta p_{i}\right)$.

In summary, we can draw the conclusion that the SE point for the Stackelberg game is unique.

In this paper, it is assumed that all FUEs are involved in the game at the beginning. In practice, the number of the FUEs is gradually reduce by iteratively removing the FUEs whose SINR requirements are not met. Next, we give an admission control algorithm to remove the FUEs which are unable to meet their requirements. That is,

$$
\bar{\gamma}_{i}<\Gamma_{i}, \quad \forall i \in S .
$$

In this scenario, an admission control scheme is proposed to protect the performances of the active FUEs. Next, the distributed algorithm combined with the admission control in two-tier femtocell networks is proposed. The flow chart of the power allocation algorithm is given in Fig. 2.

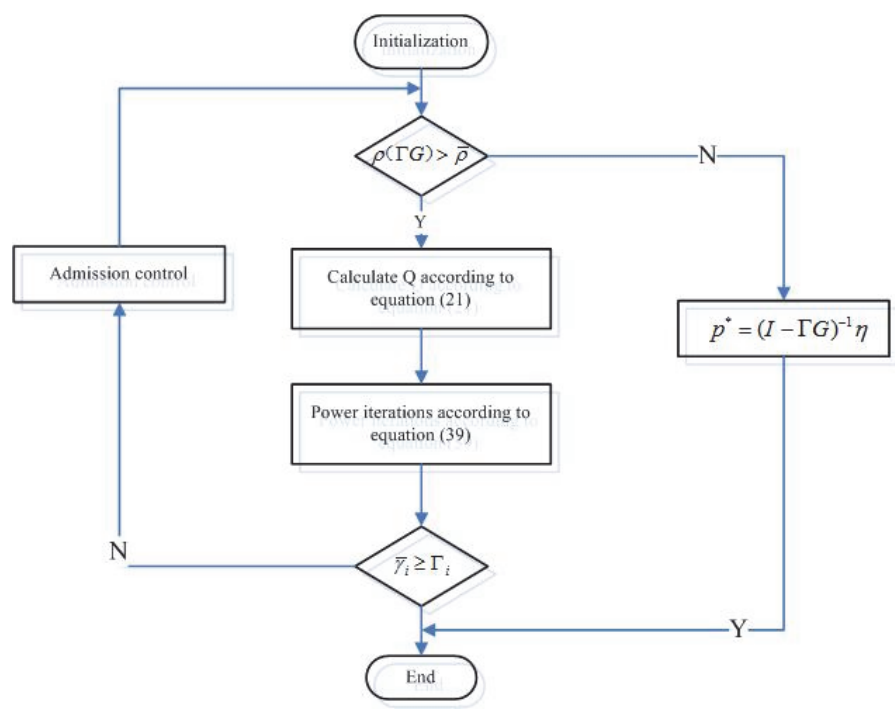

Fig. 2. Flow diagram of the power allocation algorithm. 
The pseudo codes of the proposed algorithm are given as follows.

\section{Distributed Algorithm of Power Control and Admission Control}

1 Initialize $L, \lambda_{i}, p_{i}, \bar{\rho}, \boldsymbol{\Gamma}_{f}$ and $\Gamma_{0}$.

2 The MBS measures $g_{0, i}$ and FBS $i$ measure $g_{i, j},(i, j) \in S$.

3 if $\rho(\boldsymbol{\Gamma} \boldsymbol{G})<\bar{\rho}, \boldsymbol{p}^{*}=(\boldsymbol{I}-\boldsymbol{\Gamma} \boldsymbol{G})^{-1} \boldsymbol{\eta}$ else

4 The MBS calculates $Q$ and optimal price $c^{*}$ according to equation (21) and (37), respectively.

5 Each FUE updates its transmission power by equation (39).

6 Set $\mathrm{k}=\mathrm{k}+1$, go to step 4 and repeat until convergence.

7 Each FUE calculates its normalized SINR $\bar{\gamma}_{i}$.

8 if $\bar{\gamma}_{i}<\Gamma_{i}$, FUE $i$ removes itself from the game. The remaining FUEs go to step 3 and repeat until all active users can meet the SINR requirements.

9 end.

Remark 2: In the distributed power control algorithm, there exists the case that more than one FUEs can not satisfy the SINR requirements simultaneously. The greedy strategy is used to guarantee all the other FUEs' performances. Therefore, the FUEs satisfying Item 8 in the algorithm will be simultaneously removed from the game.

\section{Simulations Results and Analysis}

In this section, numerical results are provided to illustrate the effectiveness of the proposed algorithms with the system parameters in Table I. The topology of the network used in these examples is shown in Fig. 3, where the MUE and the FUEs are randomly distributed inside circles of radius $R_{c}$ and $R_{f}$, respectively.

TABLE I

SIMULATION PARAMETERS

\begin{tabular}{c|c|c}
\hline Parameter & Variable & Value \\
\hline Macrocell radius & $R_{c}$ & $500 \mathrm{~m}$ \\
\hline Femtocell radius & $R_{f}$ & $30 \mathrm{~m}$ \\
\hline Processing gain & $L$ & 128 \\
\hline Carrier Frequency & $f_{M H z}$ & $2000 \mathrm{MHZ}$ \\
\hline Maximum Power & $p_{\max }$ & $0.5 \mathrm{~W}$ \\
\hline Required SINR of the MBS & $\Gamma_{c}$ & $\left(3, \frac{1}{q_{c}^{T} \Gamma_{f} q_{f}}\right) \mathrm{dB}$ \\
\hline Required SINRs of FUEs & $\Gamma_{f}$ & $(5,25) \mathrm{dB}$ \\
\hline Outdoor loss & $K_{f o}$ & $30 \log _{10}\left(f_{c, M H z}\right)-71 \mathrm{~dB}$ \\
\hline Indoor loss & $K_{f i}$ & $37 \mathrm{~dB}$ \\
\hline Indoor and outdoor path loss exponents & $\alpha_{c}, \alpha_{\beta}, \alpha_{f o}$ & $4,3,4$ \\
\hline Background noise & $\delta^{\prime 2}$ & $10^{-6}$ \\
\hline Partition loss & $W$ & $5 \mathrm{~dB}$ \\
\hline FUE's rate parameter & $\lambda_{i}$ & 1 \\
\hline The spectral radius & $\bar{\rho}$ & 0.99
\end{tabular}




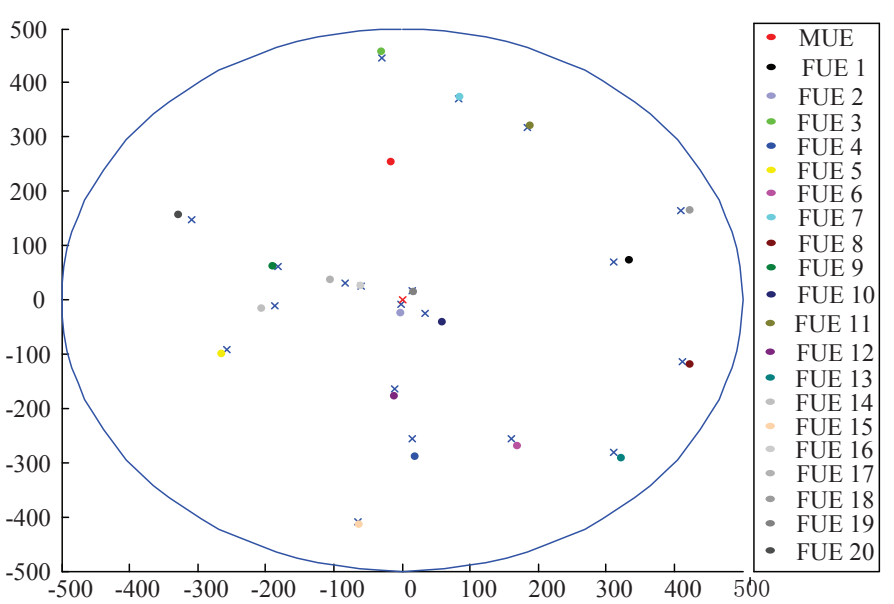

Fig. 3. Topology of the system $(n=20)$.

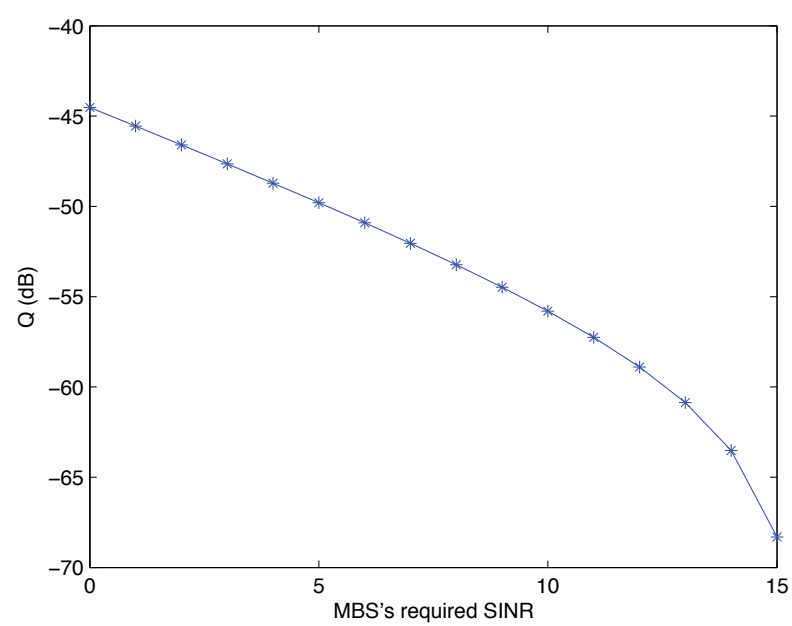

Fig. 4. Maximum tolerable interference margin $Q$ versus the MBS's required SINR.

In Fig. 4, we can see that the tolerable interference threshold is declined with the increasing of the MBS's required SINR. Obviously, it is true due to the fact that $Q=\frac{p_{0} g_{0,0}}{\Gamma_{0}}-\delta^{2}$. Therefore, in the practical transmission environment, it is necessary for MBS to set a smaller interference threshold to guarantee its own QoS requirement.

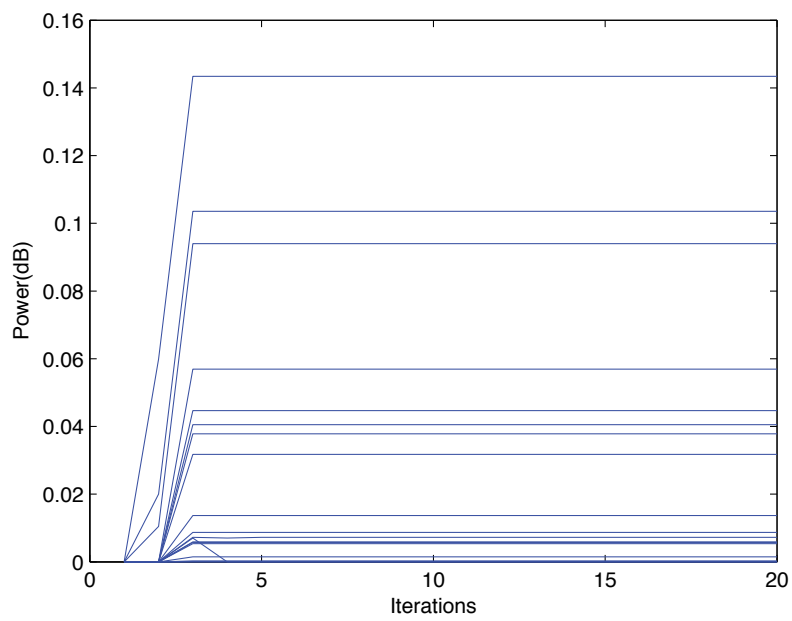

Fig. 5. Convergence performance of the power control algorithm $n=20$. 
Next we select the system with 20 FUEs, and all the initial powers are set to be 0W. It can be seen from the simulation results in Fig. 5 that the powers converge in few iterations and the convergence powers are relative small. It shows that the power control algorithm is effective. Meanwhile, the QoS requirement of the MBS can be guaranteed with the condition that the interference is not beyond the interference threshold. Next, the relationship between the interference price and the maximum tolerable interference will be investigated.

In Fig. 6, the relationship between the optimal interference prices and interference threshold is investigated. Firstly, it can be seen that, $c^{*}$ is the highest when $n=15$ and when $n=5, c^{*}$ is the lowest with the same $Q$. This is because that the competition becomes more intense with the increasing of the active FUEs. Besides, the interference prices for FUEs decrease with the increasing of $Q$. Intuitively, it can be explained by the equation $c^{*}=\frac{\boldsymbol{G}_{0} \boldsymbol{G}_{f}^{-1} \boldsymbol{A}}{\boldsymbol{G}_{0} \boldsymbol{G}_{f}^{-1} \boldsymbol{B}+Q}$. We can find that when the interference value $Q$ increases, the price $c$ will decrease. Meanwhile, we can see that the differences between the interference prices are also reduced with the increasing of $Q$.

In Fig. 7, we investigate the FUEs' average power versus interference threshold and different numbers of FUEs. It is clear that a higher average power can be obtained with the higher $Q$ as well as less number of FUEs, while the reverse is generally true. Therefore, we can get that selecting a smaller $Q$ contributes to the protection of the MBS's QoS performance.

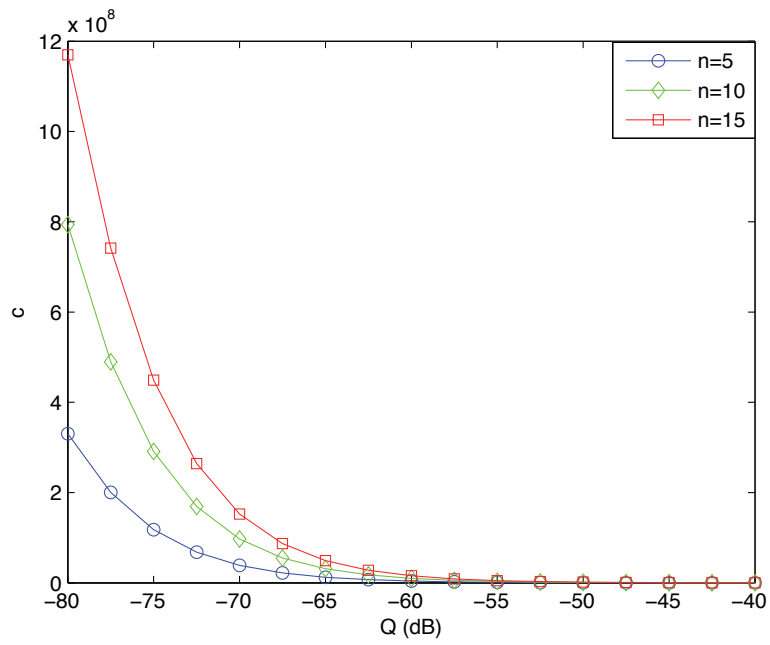

Fig. 6. The optimal interference price $c^{*}$ for FUEs versus $\mathrm{Q}$ with different number of FUEs.

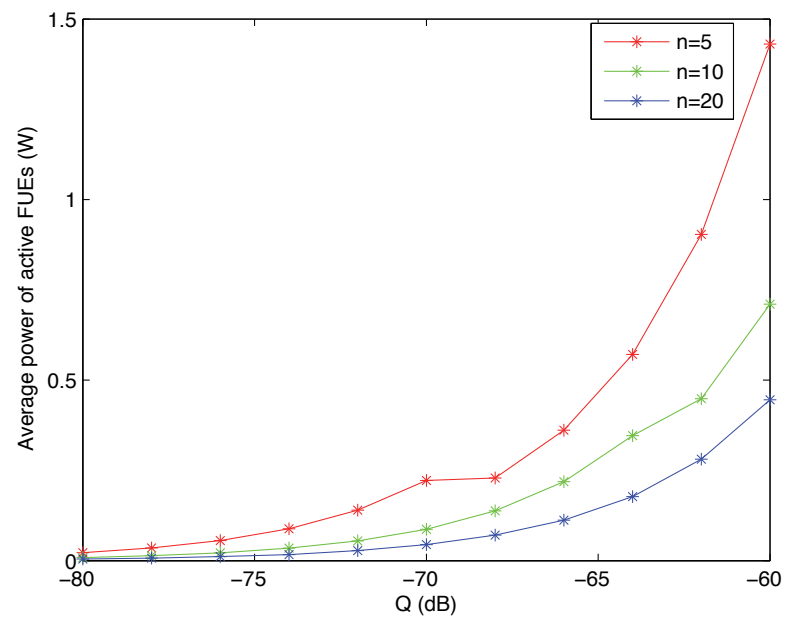

Fig. 7. The average power of active FUEs versus $Q$ with different number of FUEs.

Fig. 8(a) and Fig. 8(b) present the average SINR and average power consumption of per FUE, respectively, versus number of the FUEs, with the proposed Stackelberg game and most selfish power allocation scheme. It is worth noting that Fig. 8(a) and Fig. 8(b) show the average performance under large amounts of stochastic simulations. In 


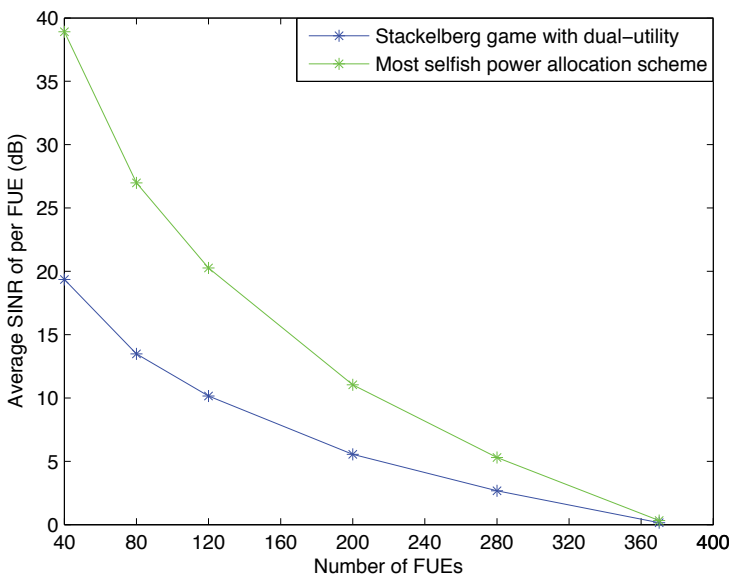

(a) Average SINR per FUE with different number of FUEs.

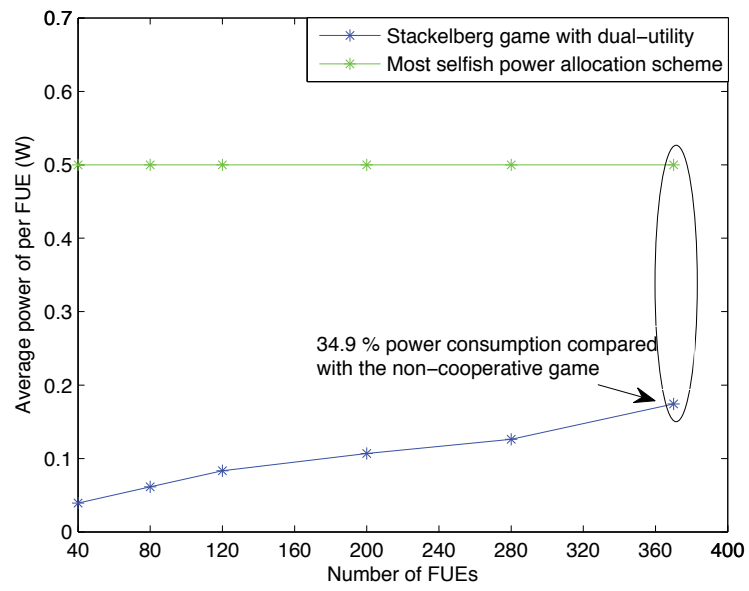

(b) Average power per FUE with different number of FUEs.

Fig. 8 Performance comparison with the most selfish power allocation scheme.

this paper, the most selfish power allocation scheme is defined through iterative process where all FUEs transmit at their maximum power $p_{\max }$. Firstly, it is observed from Fig. 8(a), the average SINR of FUEs under the most selfish power allocation scheme is higher than that under the proposed Stackelberg game. Besides, the differences between the average SINR performance decrease with the increasing of the FUEs' number. Secondly, we can see from Fig. 8(b) that the power consumption of the Stackelberg game increases with the increment of amount of the FUEs. But, the power consumption of the Stackelberg game is obviously lower than that under the most selfish power allocation scheme. For example, the FUEs' average power consumption for the proposed game is just $34.9 \%$ of the most selfish power allocation scheme's power consumption, when there are 370 FUEs in the environment.Thirdly, for the most selfish power allocation scheme, when the FUEs' number is small, it is observed from Fig. 8(a) that the average SINR performance is relatively high. However, the higher average SINR is obtained at the expense of larger power consumption which is shown in Fig. 8(b). On the other hand, when the FUEs' number is large, the average SINR performance obtained with smaller power consumption under the Stackelberg game is extremely close to that under the most selfish power allocation scheme. Then, we can get that the proposed Stackelberg game is obviously superior to the most selfish power allocation scheme in the densely scenario. At last, we can also observed from Fig. 8(a) that the proposed femtocell networks can accommodate about 370 FUEs.

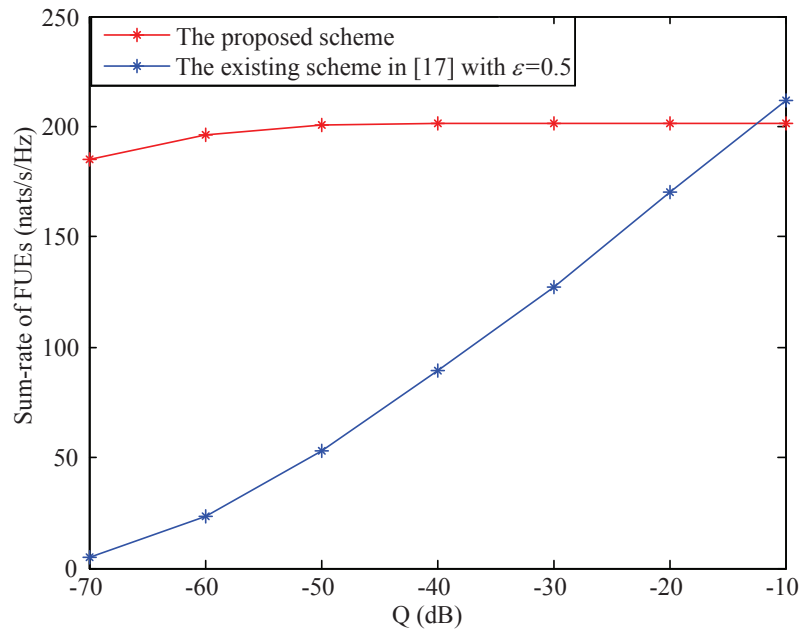

Fig. 9. Sum-rate comparison with the existing scheme.

Let us define sum-rate as $\sum_{i=1}^{N} \lambda_{i} \log \left(1+\gamma_{i}\right), i \in S$. Fig. 9 presents the sum-rate comparison results with the existing power control algorithm in [18]. In general, we can see that the sum-rate of the FUEs under the existing 
scheme is lower than that under the proposed scheme. It is because that the set of the co-tier interference boundary in the existing scheme suppresses the growth of the power. Besides, when the interference threshold is nearly $10 \mathrm{~dB}$, the sum-rate performance of the existing scheme is superior to the proposed scheme. This is because that the existing scheme does not fully consider the influence between the FUEs when $Q$ is relatively large. Therefore, we can see that the proposed power control algorithm is more suitable for application in the dynamic network environment.

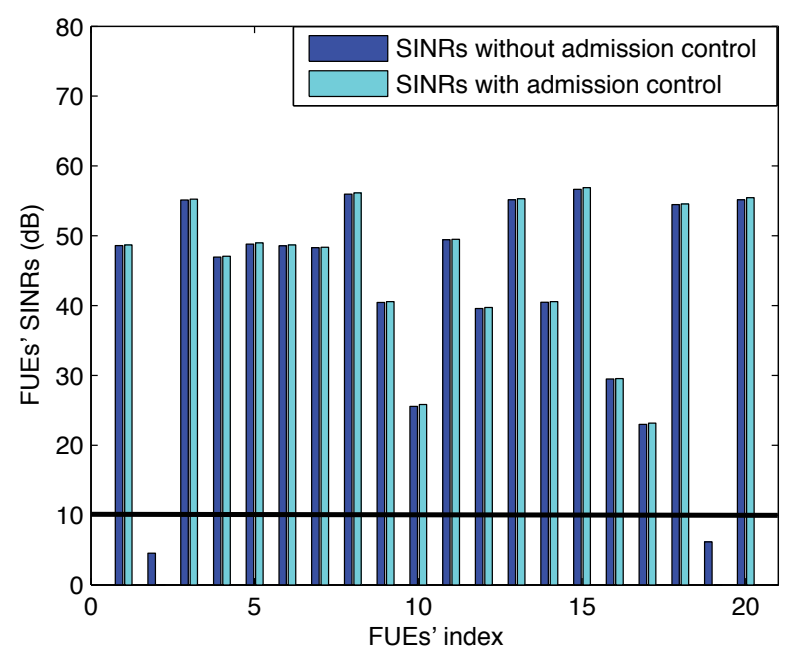

Fig. 10. SINRs of all FUEs without and with admission control.

In the simulation, the minimum SINR requirements of all FUEs are set as $\Gamma_{i}=10 \mathrm{~dB}, \forall i \in S$. In Fig. 10, we present the results of all FUEs' SINRs without and with admission control. From Fig. 10, we can get that FUE 2 and FUE 19 can not satisfy the minimum SINR requirements. Then, they will be removed from the game simultaneously. Combining with the admission control scheme, we can see that all the active FUEs' SINRs have different levels of improvement. Comparison results show the effectiveness of the proposed admission control scheme.

\section{CONCLUSION}

In this paper, we propose resource allocation strategies for two-tier femtocell networks. To analyze the interference effects among users with universal frequency reuse, the conditions that any feasible set of users' SINR requirements should satisfy are derived. In the transmission of a two-tier femtocell network, it is difficult to meet all the SINR requirements of FUEs. In this case, we formulate a Stackelberg game to jointly study the utility maximization of the macrocell and femtocells. To alleviate the cross-tier interference at the MBS, we propose a distributed power control algorithm. At last, admission control is introduced. Numerical results show that the proposed scheme can reduce the power consumption and it is more suitable for application in the dense femtocell networks. However, in this paper, the perfect channel information is assumed and the users can get accurate channel state information. The robust power allocation scheme should be consider further.

\section{ACKNOWLEDGMENT}

This work was supported in part by the National Science Foundation of China (Project numbers 61473247, 61573301, 61573303, 61571387).

\section{REFERENCES}

[1] Z. Liu, J. Wang, Y. Xia, R. Fan, H. Jiang, and H. Yang, "Power allocation robust to time-varying wireless channels in femtocell networks," IEEE Transactions on Vehicular Technology, vol. 65, no. 4, pp. 2806-2815, 2016.

[2] X. Ping, V. Chandrasekhar and J. G. Andrews, "Open vs. closed access femtocells in the uplink," IEEE Transactions on Wireless Communications, vol. 9, no. 12, pp.3798-3809, 2010.

[3] S. He, J. Chen, X. Li, X. Shen and Y. Sun, "Mobility and intruder prior information improving the barrier coverage of sparse sensor networks", IEEE Transactions on Mobile Computing, vol.13, no.6, pp.1268-1282, 2014. 
[4] H.-S. Jo, C. Mun, J. Moon and J.-G. Yook, "Interference mitigation using uplink power control for two-tier femtocell networks," IEEE Transactions on Wireless Communications, vol. 8, no. 10, pp. 4906-4910, 2009.

[5] Y. Jeong, T. Q. S. Quek and H. Shin, " Beamforming optimization for multiuser two-tier networks," Journal of Communications and Networks, vol. 13, no. 3, pp. 327-338, 2011.

[6] C.-H. Ko and H.-Y. Wei, "On-demand resource-sharing mechanism design in two-tier OFDMA femtocell networks," IEEE Transactions on Vehicular Technology, vol. 60, no. 3, pp. 1059-1071, 2011.

[7] S.-M. Cheng, S.-Y. Lien, "On exploiting cognitive radio to mitigate interference in macro/femto heterogeneous networks," IEEE Transactions on Wireless Communications, vol. 18, no. 3, pp. 40-47, 2011.

[8] R. Madan, J. Borran, A. Sampath, N. Bhushan, A. Khandekar and T. Ji, "Cell association and interference coordination in heterogeneous LTE-A cellular networks," IEEE Journal on Selected Areas in Communications, vol. 29, no. 9, pp. 1479-1489, 2010.

[9] M. Maso, M. Debbah and L. Vangelista, "A distributed approach to interference alignment in OFDM-based two-tier networks," IEEE Transactions on Vehicular Technology, vol. 62, no. 5, pp. 1935-1949, 2013.

[10] G. J. Foschini and Z. Miljanic, "A simple distributed autonomous power control algorithm and its convergence," IEEE Transactions on Vehicular Technology, vol. 42, no. 4, pp. 641-646, 1993.

[11] D. T. Ngo, L. B. Le, L. N. THO, E. Hossain and D. I. Kim, "Distributed interference management in two-tier CDMA femtocell networks," IEEE Transactions on Wireless Communications, vol. 11, no. 3, pp. 979-989, 2012.

[12] Z. Huang, Z. M. Zeng and H. Xia, "Hierarchical power game with dual-utility in two-tier OFDMA femtocell networks," International Conference on Wireless Communications, Networking and Mobile Computing, pp. 23-25, 2011.

[13] X. Kang, Y. Liang and H. K. Garg, "Distributed power control for spectrum-sharing femtocell networks using Stackelberg game," IEEE International Conference on Communications, pp. 5-9, 2011.

[14] G. Cao, D. Yang, X. Ye and X. Zhang, "A downlink joint power control and resource allocation scheme for co-channel macrocellfemtocell networks," 2011 IEEE Wireless Communication and Networking Conference, DOI:10.1109/WCNC.2011.5779141, pp. 281286, 2011.

[15] Z. Lu, T. Bansal and P. Sinha, "Achieving user-level fairness in open-access femtocell based architecture," IEEE Transactions on Mobile Computing, vol. 12, no. 10, pp. 1943-1954, 2013.

[16] D. Kim, E. Shin and M. Jin, "Hierarchical power control with interference allowance for uplink transmission in two-tier heterogeneous network," IEEE Transactions on Wireless Communications, vol. 14, no. 2, pp. 616-627, 2015.

[17] X. Kang, R. Zhang and M. Motani, "Price-based resource allocation for spectrum-sharing femtocell networks: A Stackelberg game approach," IEEE Journal on Selected Areas in Communications, vol. 30, no. 3, pp. 538-549, 2012.

[18] J. Zhang, Z. Zhao, W. Li, Z. Liu and L. Xin, "Joint pricing and power control for OFDMA femtocell networks using Stackelberg game," International Symposium on Wireless Personal Multimedia Communications, pp. 619-622, 2012

[19] R. Estrada, A. Jarray, H. Otrok, Z. Dziong, H. Barada, "Energy-efficient resource-allocation model for OFDMA macrocell/femtocell networks," IEEE Transactions on Vehicular Technology, vol. 62, no. 7, pp. 3429-3437, 2013.

[20] D. I. Kim, E. H. Shin and M. S. Jin, "Hierarchical power control with interference allowance for Uplink transmission in two-tier heterogeneous networks," IEEE Transactions on Wireless Communications, vol. 14, no. 2, pp. 616-627, 2015.

[21] P. Hande, S. Rangan, M. Chiang and X. Wu, "Distributed uplink power control for optimal SIR assignment in cellular data networks," IEEE/ACM Transactions on Networking, vol. 16, no. 6, pp. 1420-1433, 2008.

[22] T. N. Duy, B. L. Long and T. Le-Ngoc, "Distributed Pareto-optimal power control for utility maximization in femtocell networks," IEEE Transactions on Wireless Communications, vol. 11, no. 10, pp. 3434-3446, 2012.

[23] V. Chandrasekhar, J. G. Andrews, T. Muharemovic and A. Gatheerer, "Power control in two-tier femtocell networks," IEEE Transactions on Wireless Communications, vol. 8, no. 8, pp. 4316-4328, 2009.

[24] C. Meyer, "Uncoupling the Perron eigenvector problem," Liner Algerbra and its applications, vol. 114, no. 115, pp. 69-94, 1989.

[25] H. Claussen, L. Ho and L. Samuel, "Self-optimization of coverage for femtocell deployments," Wireless Telecommunications Symposium, pp. 278-285, 2008. 